\title{
An autosomal recessive syndrome of severe mental retardation, cataract, coloboma and kyphosis maps to the pericentromeric region of chromosome 4
}

\author{
Kimia Kahrizi ${ }^{1}$, Hossein Najmabadi ${ }^{1}$, Roxana Kariminejad ${ }^{1}$, Payman Jamali ${ }^{1}$, \\ Mahdi Malekpour ${ }^{1}$, Masoud Garshasbi ${ }^{1,2}$, Hans Hilger Ropers ${ }^{2}$, Andreas Walter Kuss ${ }^{2}$ and \\ Andreas Tzschach*,2
}

${ }^{1}$ Genetics Research Center, University of Social Welfare and Rehabilitation Sciences, Tehran, Iran; ${ }^{2}$ Department Human
Molecular Genetics, Max Planck Institute for Molecular Genetics, Berlin, Germany

We report on three siblings with a novel mental retardation (MR) syndrome who were born to distantly related Iranian parents. The clinical problems comprised severe MR, cataracts with onset in late adolescence, kyphosis, contractures of large joints, bulbous nose with broad nasal bridge, and thick lips. Two patients also had uni- or bilateral iris coloboma. Linkage analysis revealed a single $10.4 \mathrm{Mb}$ interval of homozygosity with significant LOD score in the pericentromeric region of chromosome 4 flanked by SNPs rs728293 (4p12) and rs1105434 (4q12). This interval contains more than 40 genes, none of which has been implicated in MR so far. The identification of the causative gene defect for this syndrome will provide new insights into the development of the brain and the eye.

European Journal of Human Genetics (2009) 17, 125-128; doi:10.1038/ejhg.2008.159; published online 10 September 2008

Keywords: mental retardation; autosomal recessive; consanguinity; cataract; coloboma; kyphosis

\section{Introduction}

Mental retardation (MR) has a prevalence of about $2 \%,{ }^{1}$ and more than $50 \%$ of patients with moderate-to-severe MR are thought to have genetic defects. ${ }^{2}$ As part of a large collaborative project to identify genes involved in autosomal recessive $\mathrm{MR}^{3}{ }^{3}$ we performed linkage analysis in an Iranian family with three affected siblings who suffered from an apparently novel syndromic form of MR associated with cataract, coloboma and kyphosis. Here, we report on the clinical details of the patients and the identification of a gene locus in the pericentromeric region of chromosome 4 .

*Correspondence: Dr A Tzschach, Department Human Molecular Genetics, Max Planck Institute for Molecular Genetics, Ihnestrasse 73, 14195 Berlin, Germany.

Tel: +4930 8413 1416; Fax: +49308413 1383;

E-mail: tzschach@molgen.mpg.de

Received 7 April 2008; revised 1 July 2008; accepted 30 July 2008; published online 10 September 2008

\section{Clinical report}

The pedigree of the family is shown in Figure 2a. Both parents came from the same village in central Iran and were presumably related; the precise relationship, however, could not be determined. As they had neither common grandparents nor great-grandparents, we assumed thirdcousin relationship for linkage analysis.

The parents and five of their eight children were healthy. The clinical features of the three affected siblings (II:4; II:5 and II:6) are listed in Table 1. They all suffered from severe MR, they never learned to speak, and their motor development was delayed (Figure 1a-c). Two of them had iris coloboma (unilateral in II:4 and bilateral in II:6), and all three siblings suffered from cataracts since about the age of 17 years. Starting at about 8 years of age, all three siblings developed thoracic kyphosis (Figure 1d), but skeletal X-rays revealed no vertebral abnormalities. They also developed contractures of the knee and elbow joints. Neither seizures nor other neurological problems had been reported. Body 
Table 1 Clinical features of the patients

\begin{tabular}{|c|c|c|c|}
\hline Clinical feature & Patient II:4 & Patient II:5 & Patient II:6 \\
\hline Sex & Male & Female & Male \\
\hline Age of examination (years) & 45 & 42 & 40 \\
\hline Mental retardation & Severe & Severe & Severe \\
\hline Body height $(\mathrm{cm})$ & 148 ( $\ll 3$ rd cent. $)$ & 138 ( $\ll 3$ rd cent. $)$ & 145 ( $\ll 3$ rd cent. $)$ \\
\hline Weight $(\mathrm{kg})$ & 49 & 47 & 51 \\
\hline Head circumference $(\mathrm{cm})$ & 54 (10th cent.) & 51 (<3rd cent.) & 52 (<3rd cent.) \\
\hline Kyphosis & + & + & + \\
\hline Contractures of knees and elbows & + & + & + \\
\hline \multicolumn{4}{|l|}{ Facial dysmorphisms } \\
\hline Thick lips & + & + & + \\
\hline Bulbous nose & + & + & + \\
\hline Broad nasal bridge & + & + & + \\
\hline \multicolumn{4}{|l|}{ Ocular abnormalities } \\
\hline Cataract & Bilateral & Bilateral & Bilateral \\
\hline Iris coloboma & Left iris & - & Bilateral \\
\hline Other problems & Facial haemangioma (left) & - & - \\
\hline
\end{tabular}
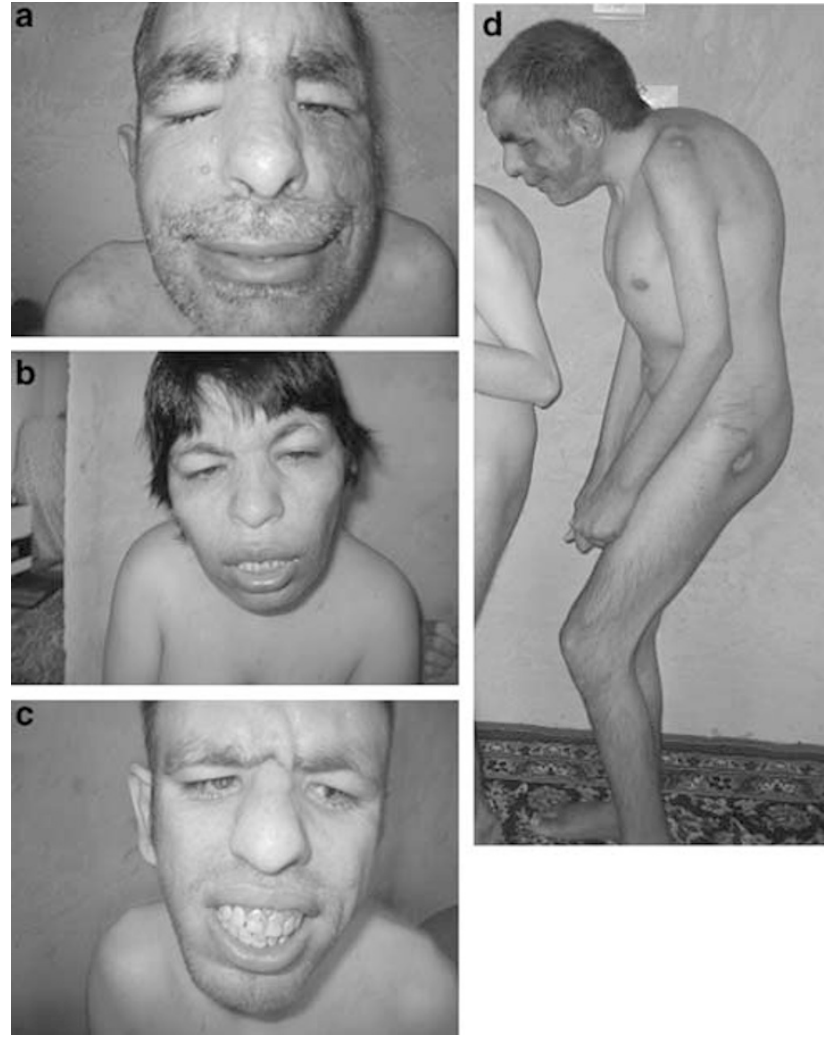

Figure 1 (a-c) Facial features of patients II:2 (a), II:3 (b) and II:4 (c). Note bulbous nose, broad nasal bridge and thick lips in all patients; and a large haemangioma on the left cheek of patient II:2 (a); and bilateral iris coloboma in patient II:4 (c). (d) Patient II:2. Note kyphosis and contractures of elbows and knees.

heights were short, but also their parents (mother $150 \mathrm{~cm}$ and father $160 \mathrm{~cm}$ ) and their healthy siblings had short stature. Facial features in all affected siblings included thick lips, bulbous nose and broad nasal bridge; and the oldest brother (II:4) had a large capillary haemangioma on his left cheek.

A brain CT scan of patient II:4 at the age of 36 years revealed no abnormalities. Chromosome analysis on peripheral blood lymphocytes of patient II:4 revealed a normal male karyotype 46,XY (GTP banding, 450 bands). Fragile X-testing and metabolic screening by tandem mass spectroscopy in patient II:4 were normal.

\section{Materials and methods}

DNA was extracted from peripheral blood of the patients (II:4, II:5, and II:6), two healthy siblings (II:1 and II:3) and their parents (I:1 and I:2) using standard procedures after informed consent (Figure 2a). Genotyping (SNP analysis) was performed using the Human Mapping 10k Array, Version 2 (Affymetrix) ${ }^{4}$ based on previously published protocols. ${ }^{5}$ Linkage analysis was performed using the Merlin software. Details of data quality controls and linkage analysis have been published elsewhere. ${ }^{6}$

\section{Results}

Linkage analysis in this family revealed a single interval of homozygosity in the pericentromeric region of chromosome 4 (4p12-4q12) with significant LOD scores of 3.38 in parametric analysis and LOD 4.53 in non-parametric analysis assuming third-cousin relationship of the parents (Figure $2 \mathrm{~b}$ and $\mathrm{c}$ ). The linkage interval had a size of $10.4 \mathrm{Mb}$ and was flanked by the heterozygous SNPs rs728293 (chr4: 47052440, UCSC genome browser March 2006 assembly) and rs1105434 (chr4: 57488508, UCSC genome browser March 2006 assembly) (Figure 2a). The interval harbours the following 46 RefSeq genes: GABRB1, REST, NMU, 
a

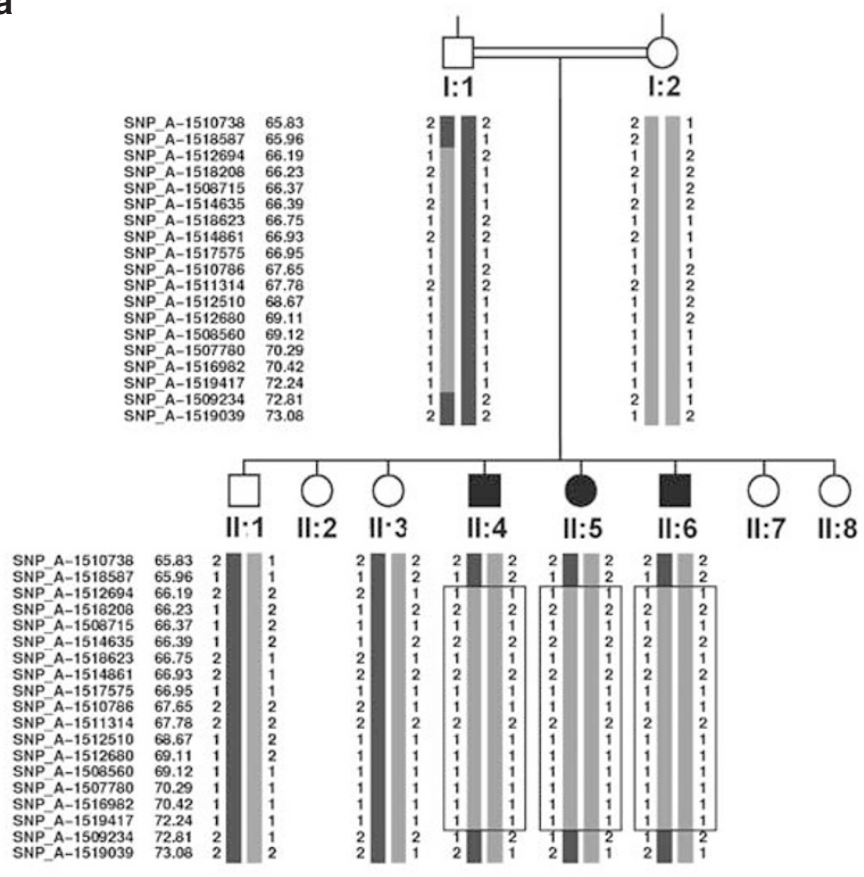

b
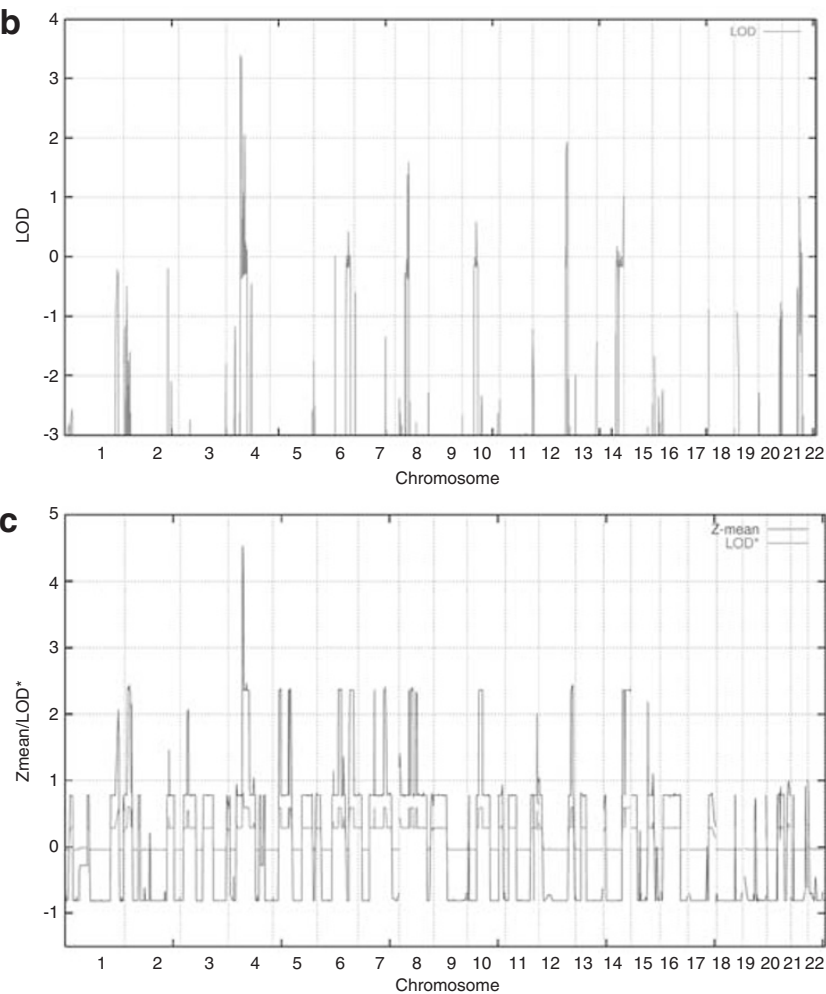

Figure 2 (a) Pedigree of the family and haplotypes of the linkage interval on chromosome 4. (b and c) Results of the parametric (b) and nonparametric (c) linkage analyses using the Merlin software. Both analyses revealed a single linkage interval in the pericentromeric region of chromosome 4 , with LOD scores of 3.38 (parametric analysis) and 4.53 (non-parametric analysis) assuming third-cousin relationship of the parents.

AASDH, CORIN, KIT, OCIAD2, PDGFRA, CNGA1, CEP135, CHIC2, CLOCK, COMMD8, EXOC1, SRP72, SCL1OA4, TEC, SGCB, SCFD2, RASL11B, PPAT, PDCL2, ATP1OD, DCUN1D4, FLJ21511, FIP1L1, KDR, NPAL1, OCIAD1, PAICS, LOC402176, USP46, NFXL1, SPATA18, TXK, ZAR1, LNX1, SPINK2, ARL9, SLAIN2, LRRC66, GSX2, KIAA1211, HOPX, SRD5A and TMEM165.

\section{Discussion}

The three siblings of this family shared nearly identical clinical features comprising severe MR, cataracts with onset in late adolescence, kyphosis, contractures of large joints, bulbous nose with broad nasal bridge, and thick lips. Iris coloboma seems to be a variable feature: whereas coloboma in the youngest brother (II:6) was bilateral, it was unilateral in the eldest brother (II:4), and there was no coloboma in the sister (II:5).

Phenotypically, the family overlaps with several known MCA (multiple congenital anomalies)/MR syndromes. Iris coloboma can be found in patients with autosomal recessive Peters plus syndrome (OMIM 261540), which is characterized by variable ocular and extraocular features.
A gene for Peters plus syndrome (B3GALTL) is located on chromosome 13. ${ }^{7}$ Baraitser and Winter ${ }^{8}$ reported a (possibly autosomal recessive) syndrome of coloboma, ptosis, hypertelorism and MR (OMIM 243310), but these patients had no cataract. Mutations in CHX10 on chromosome 14 cause autosomal recessive microphthalmia with coloboma and cataract (OMIM 610092); these patients have, however, normal mental development. ${ }^{9}$ Coloboma is also a feature of CHARGE syndrome (OMIM 214800) and Cat eye syndrome (OMIM 115470), but these syndromes are characterized by numerous additional problems, which are not present in this family.

Cataract, kyphoscoliosis and MR, but not coloboma, are features of Warburg Micro syndrome (OMIM 600118), an autosomal recessive disorder caused by mutations in RAB3GAP on chromosome $2 .^{10}$ These features are also found in the closely related Martsolf syndrome (OMIM 212720), which is caused by RAB3GAP2 mutations (chromosome 1). ${ }^{11}$

However, none of the syndromes mentioned above fits convincingly the siblings reported here. We therefore presume that these patients suffer from a new autosomal recessive MCA/MR syndrome. This assumption is supported by the identification of a gene locus in the pericentromeric 
region of chromosome 4 . The linkage interval harbours more than 40 genes, three of which - KIT, SGCB and KDR had been implicated in human disorders before. Defects of KIT cause autosomal dominant piebaldism (OMIM 172800), ${ }^{12}$ and mutations in $S G C B$, which encodes beta-sarcoglycan, cause autosomal recessive limb-girdle muscular dystrophy LGMD2E (OMIM 604286). ${ }^{13}$ As one of our patients (II:4) had a large facial haemangioma, it is noteworthy that a somatic missense mutation of KDR (VEGFR2 and FLK1) has been reported in a capillary haemangioma sample, ${ }^{14}$ but a causal link between this gene and the severe clinical problems of this family is not very likely.

Genotyping other families or single patients with a similar combination of clinical features may reduce the size of the linkage interval and thus facilitate the identification of the causative gene defect(s). This will not only be a starting point to elucidate the underlying pathomechanisms of this disorder but will also eventually provide further insights into the development of the brain and the eye.

\section{Acknowledgements}

We are grateful to the patients and the parents of the family for their cooperation. We thank Bettina Lipkowitz for technical assistance. Our study was supported by the Iranian Molecular Medicine Network (HN), the Max Planck Innovation Fund (H-HR) and the DFG SFB 577 project $(H-H R)$.

\section{References}

1 Leonard $\mathrm{H}$, Wen X: The epidemiology of mental retardation: challenges and opportunities in the new millennium. Ment Retard Dev Disabil Res Rev 2002; 8: 117-134.
2 van Karnebeek CD, Scheper FY, Abeling NG et al: Etiology of mental retardation in children referred to a tertiary care center: a prospective study. Am J Ment Retard 2005; 110: 253-267.

3 Najmabadi H, Motazacker MM, Garshasbi M et al: Homozygosity mapping in consanguineous families reveals extreme heterogeneity of non-syndromic autosomal recessive mental retardation and identifies 8 novel gene loci. Hum Genet 2007; 121: 43-48.

4 Kennedy GC, Matsuzaki H, Dong S et al: Large-scale genotyping of complex DNA. Nat Biotechnol 2003; 21: 1233-1237.

5 Matsuzaki H, Loi H, Dong $\mathrm{S}$ et al: Parallel genotyping of over 10000 SNPs using a one-primer assay on a high-density oligonucleotide array. Genome Res 2004; 14: 414-425.

6 Garshasbi M, Motazacker MM, Kahrizi K et al: SNP array-based homozygosity mapping reveals MCPH1 deletion in family with autosomal recessive mental retardation and mild microcephaly. Hum Genet 2006; 118: 708-715.

7 Lesnik Oberstein SA, Kriek M, White SJ et al: Peters Plus syndrome is caused by mutations in B3GALTL, a putative glycosyltransferase. Am J Hum Genet 2006; 79: 562-566.

8 Baraitser M, Winter RM: Iris coloboma, ptosis, hypertelorism, and mental retardation: a new syndrome. J Med Genet 1988; 25: $41-43$.

9 Bar-Yosef U, Abuelaish I, Harel T, Hendler N, Ofir R, Birk OS: CHX10 mutations cause non-syndromic microphthalmia/anophthalmia in Arab and Jewish kindreds. Hum Genet 2004; 115: 302-309.

10 Aligianis IA, Johnson CA, Gissen P et al: Mutations of the catalytic subunit of RAB3GAP cause Warburg Micro syndrome. Nat Genet 2005; 37: 221-223.

11 Aligianis IA, Morgan NV, Mione $\mathrm{M}$ et al: Mutation in Rab3 GTPase-activating protein (RAB3GAP) noncatalytic subunit in a kindred with Martsolf syndrome. Am J Hum Genet 2006; 78: $702-707$.

12 Giebel LB, Spritz RA: Mutation of the KIT (mast/stem cell growth factor receptor) protooncogene in human piebaldism. Proc Natl Acad Sci USA 1991; 88: 8696-8699.

13 Lim LE, Duclos F, Broux O et al: Beta-sarcoglycan: characterization and role in limb-girdle muscular dystrophy linked to $4 \mathrm{q} 12$. Nat Genet 1995; 11: 257-265.

14 Walter JW, North PE, Waner M et al: Somatic mutation of vascular endothelial growth factor receptors in juvenile hemangioma. Genes Chromosomes Cancer 2002; 33: 295-303. 